University of Texas at El Paso

ScholarWorks@UTEP

$12-2010$

\title{
Fundamental Physical Equations Can Be Derived By Applying Fuzzy Methodology to Informal Physical Ideas
}

\author{
Eric Gutierrez \\ The University of Texas at El Paso, ejgutierrez@miners.utep.edu \\ Vladik Kreinovich \\ The University of Texas at El Paso, vladik@utep.edu
}

Follow this and additional works at: https://scholarworks.utep.edu/cs_techrep

Part of the Computer Engineering Commons

Comments:

Technical Report: UTEP-CS-10-37a

Published in Proceedings of the 30th Annual Conference of the North American Fuzzy

Information Processing Society NAFIPS'2011, El Paso, Texas, March 18-20, 2011.

\section{Recommended Citation}

Gutierrez, Eric and Kreinovich, Vladik, "Fundamental Physical Equations Can Be Derived By Applying Fuzzy Methodology to Informal Physical Ideas" (2010). Departmental Technical Reports (CS). 670.

https://scholarworks.utep.edu/cs_techrep/670

This Article is brought to you for free and open access by the Computer Science at ScholarWorks@UTEP. It has been accepted for inclusion in Departmental Technical Reports (CS) by an authorized administrator of ScholarWorks@UTEP. For more information, please contact Iweber@utep.edu. 


\title{
Fundamental Physical Equations Can Be Derived By Applying Fuzzy Methodology to Informal Physical Ideas
}

\author{
Eric Gutierrez and Vladik Kreinovich \\ Department of Computer Science, University of Texas at El Paso \\ 500 W. University, El Paso, TX 79968, USA \\ ejgutierrez@miners.utep.edu, vladik@utep.edu
}

\begin{abstract}
Fuzzy methodology transforms expert ideas - formulated in terms of words from natural language - into precise rules and formulas. In this paper, we show that by applying this methodology to intuitive physical ideas, we can get fundamental physical equations. This fact provides an additional justification for the fuzzy methodology.
\end{abstract}

\section{INTRODUCTION}

Fuzzy methodology: main objective. Fuzzy methodology has been invented to transform expert ideas - formulated in terms of words from natural language - into precise rules and formulas, rules and formulas understandable by a computer and implementable on a computer; see, e.g., [3], [4].

Fuzzy methodology: main success. Up to now, the main success - and thus, the main confirmation - of fuzzy methodology was in intelligent (fuzzy) control [3], [4].

What we plan to do. In this paper, we show that the same fuzzy methodology can also lead - when applied appropriately - to the exact fundamental equations of physics. This fact provides an additional justification for the fuzzy methodology.

\section{CASE Study: Newton's Physics}

Newton's physics: informal description. Let us consider a simple case when we have a single body in a potential field $V(x)$. It is a commonsense knowledge that a body usually tries to go to the points $x$ where its potential energy $V(x)$ is the smallest. For example, a rock left at the top of the mountain, when it starts moving, it may sometimes move up (due to the original push), but mostly it tries to go down.

If we take friction into account, then a body also tries to stop. In the idealized case when there is no friction, there is a conservation of energy: the sum of the potential energy $V(x)$ and the kinetic energy

$$
K=\frac{1}{2} \cdot m \cdot \sum_{i=1}^{3}\left(\frac{d x_{i}}{d t}\right)^{2}
$$

is constant. Thus, when the body minimizes its potential energy, it thus tries to maximize its kinetic energy.

What we plan to do. The above text does not sound like a very accurate description of a physical system. However, we will show that when we apply the usual fuzzy methodology to this description, we get a very precise formulation - all the way to Newton's equations

$$
m \cdot \frac{d^{2} x_{i}}{d t^{2}}=-\frac{\partial V}{\partial x_{i}} .
$$

We will perform this derivation step-by-step.

First step: selecting a physically meaningful membership function corresponding to "small $V(x)$ ". The body tries to get to the areas where the potential energy $V(x)$ is small. "Small" is an imprecise word from natural language. For such words, the fuzzy methodology recommends to select a membership function $\mu(V)$ describing, for each possible value $V$, to what extent this value $V$ is small.

How membership functions are determined: one of the possible ways. For each individual value $V$, the value $\mu(V)$ can be obtained, e.g., by polling several $(n)$ experts and assigning, as $\mu(V)$, the ratio

$$
\mu(V)=\frac{n(V)}{n},
$$

where $n(V)$ is the number of experts who answered that this value $V$ is small.

Specifics of a physical system. In assigning the appropriate membership function, we must take into account the specifics of the physical system. One of the features of this physical system (see, e.g., [2]) is that the potential energy has no absolute numerical value. All we know is the relative potential energy relative to some level. If we change that level by some value $V_{0}$, then all the numerical values of the potential energy get shifted, from $V$ to $V+V_{0}$.

Crudely speaking, this means that the numerical values $V$ and $V+V_{0}$ may represent the exact same value of the potential energy - but measured in comparison to different levels.

How to describe these physical specifics: first try. A seemingly natural formalization of this idea is to simply require that the degrees to which the values $V$ and $V+V_{0}$ are small should be the same: $\mu(V)=\mu\left(V+V_{0}\right)$.

The first try does not work. However, this formalization does not work: if we require that this equality holds for all $V$ and $V_{0}$, then for every two real numbers $V$ and $V^{\prime}$, by taking 
$V_{0}=V^{\prime}-V$, we would be able to conclude that $\mu(V)=$ $\mu\left(V+V_{0}\right)=\mu\left(V^{\prime}\right)-$ and thus, that the resulting membership function is simply constant. This does not make intuitive sense, since we know that the smaller the value $V$, the larger should be our confidence that $V$ is small.

A better idea. We therefore cannot simply require that the functions $\mu(V)$ and $\mu\left(V+V_{0}\right)$ corresponding to two different levels are identical. However, we should require that these two membership functions be, to some extent, equivalent to each other.

How to formalize this idea: re-analyzing the polling method. How can we formalize this idea? To do that, let us go back to the polling method of determining a membership function.

Our objective is to find the value $\mu(V)$ as accurately as possible. It is known that in the poll, the more people we ask, the more accurate is the resulting opinion. Thus, a natural way to improve the accuracy of the poll is to ask more experts. However, there is a catch. When at first, we could only afford to poll $n$ people, we thus selected top experts in the field. Now that we add $m$ extra folks, these folks may be too intimidated by the original experts to voice their opinions - especially in case the original experts disagree. With the new experts mute, we still have the same number $n(V)$ of experts who believe that the value $V$ is small - but now we have to divide it not by the original number $n$, but by the new number $n+m$. As a result, instead of the original value $\mu(V)=\frac{n(N)}{n}$, we get a new value $\mu^{\prime}(V)=\frac{n(N)}{n+m}$. It is easy to see that $\mu^{\prime}(V)=$ $c \cdot \mu(V)$, where $c=\frac{n+m}{n+m}$.

Thus, for the exact same opinion, by selecting two different numbers of experts $n$ and $n+m$, we get two numerically different membership functions: $\mu(V)$ and $c \cdot \mu(V)$. These two membership functions represent the same expert opinion and are, thus, equivalent in some reasonable sense.

Resulting formalization of the physical intuition. Now, we have a meaningful interpretation of the requirement that the membership functions $\mu(V)$ and $\mu\left(V+V_{0}\right)$ - corresponding to two different starting levels for measuring potential energy - are equivalent: that for every $V_{0}$, there should be a value $c\left(V_{0}\right)$ for which

$$
\mu\left(V+V_{0}\right)=c\left(V_{0}\right) \cdot \mu(V) .
$$

Resulting selection of the membership function. This functional equation is known (see, e.g., [1]). Its only monotonic solution is a function

$$
\mu(V)=a \cdot \exp (-k \cdot V) .
$$

So we will use this exponential function to describe the fact that potential energy should be small.

Comment. Since, as we have mentioned, the membership function is determined modulo a factor $c$, we can, for simplicity, set $a$ to 1 and get an even simpler formula $\mu(V)=\exp (-k \cdot V)$.

Second step: selecting a membership function corresponding to "large value of kinetic energy $K$ ". As we have mentioned, kinetic energy tends to increase, i.e., should be large.

Instead of starting a derivation from scratch, let us use the fact that we already have a physically meaningful membership function for "small". Intuitively, a value $K$ is large if $-K$ is small.

So, the statement "kinetic energy $K$ is large" is equivalent to saying "the value $-K$ is small". By using the above membership function for small, we thus conclude that the membership function describing our intuition about the kinetic energy is

$$
\mu(K)=\exp (-k \cdot(-K))=\exp (k \cdot K) .
$$

Third step: selecting a physically meaningful t-norm ("and"-operation). We want to describe the intuition that the potential energy is small and that the kinetic energy is large and that the same is true at different moments of time. According to fuzzy methodology, we must therefore select an appropriate "and"-operation (t-norm) to combine our degrees of certainty in individual statements into a single degree describing the degree to which we believe in the composite statement.

Let us use physical intuition to select such a t-norm $f_{\&}(a, b)$.

Specific of the physical system. In principle, if we have two completely independent systems, we can consider them as a single system. Since these systems do not interact with each other, the total energy $E$ of the combined system is simply equal to the sum $E_{1}+E_{2}$ of the energies of the components.

Using the physical specifics. Intuitively, if both component energies are small, then the resulting total energy should also be small. We can therefore estimate the smallness of the total energy in two different ways:

- first, we can simply apply the above membership function "small" to the total energy $E=E_{1}+E_{2}$, and get the value $\mu\left(E_{1}+E_{2}\right)$;

- second, we can first estimate the degrees $\mu\left(E_{1}\right)$ and $\mu\left(E_{2}\right)$ to which each of the components is small, and then use a t-norm $f_{\&}(a, b)$ to combine these degrees into a degree that $E_{1}$ is small and $E_{2}$ is small: $f_{\&}\left(\mu\left(E_{1}\right), \mu\left(E_{2}\right)\right)$.

In view of the above motivation, it is reasonable to require that these two estimates should coincide, i.e., that we should have

$$
\mu\left(E_{1}+E_{2}\right)=f_{\&}\left(\left(\mu\left(E_{1}\right), \mu\left(E_{2}\right)\right) .\right.
$$

We know that $\mu(E)=\exp (-k \cdot E)$, thus, we conclude that the following equality should hold for all $E_{1}$ and $E_{2}$ :

$$
\exp \left(-k \cdot\left(E_{1}+E_{2}\right)\right)=f_{\&}\left(\exp \left(-k \cdot E_{1}\right), \exp \left(-k \cdot E_{2}\right)\right) \text {. }
$$


This requirement enables us to uniquely determine the corresponding t-norm. Namely, to find the value $f_{\&}\left(a_{1}, a_{2}\right)$, we must first find the values $E_{i}$ for which $\exp \left(-k \cdot E_{i}\right)=a_{i}$. For these values, we then have

$$
\begin{gathered}
f_{\&}\left(a_{1}, a_{2}\right)=\exp \left(-k \cdot\left(E_{1}+E_{2}\right)\right)= \\
\exp \left(-k \cdot E_{1}\right) \cdot \exp \left(-k \cdot E_{2}\right)=a_{1} \cdot a_{2} .
\end{gathered}
$$

Resulting selection of a t-norm. Thus, the physically meaningful t-norm is the algebraic product $f_{\&}\left(a_{1}, a_{2}\right)=a_{1} \cdot a_{2}$.

Resulting model. Now, we are ready to estimate to what extent a given trajectory $x(t)$ satisfies the intuitive ideas that the potential energy be small and the kinetic energy be large at all moments of time $t_{1}, \ldots, t_{N}$. We know the degrees to which each of these requirements is satisfied at each moment of time, so to get the overall degree, we can simply multiply all these degrees. As a result, we get the following product:

$$
\prod_{i=1}^{N} \exp \left(-k \cdot V\left(t_{i}\right)\right) \cdot \prod_{i=1}^{N} \exp \left(k \cdot K\left(t_{i}\right)\right) .
$$

Since, as we have already mentioned,

$$
\exp (-k \cdot a) \cdot \exp (-k \cdot b)=\exp (-k \cdot(a+b)),
$$

this expression can be reformulated as $\exp (-k \cdot S)$, where

$$
S \stackrel{\text { def }}{=} \sum_{i=1}^{N}\left(V\left(t_{i}\right)-K\left(t_{i}\right)\right)
$$

It is reasonable to select, as the most reasonable, a trajectory for which our degree of confidence that this trajectory is reasonable is the highest. To find such a trajectory, we must maximize the value $\exp (-k \cdot S)$. Since the function $\exp (-k \cdot S)$ is strictly decreasing, this is equivalent to minimizing $S$.

So, we arrive at the requirement that we should minimize the sum $S$. In reality, the number of moments of time is infinite, so instead of a sum, we get an integral

$$
S \sim \int L d t
$$

where we denoted

$$
L=V(t)-K(t)=V(t)-\frac{1}{2} \cdot m \cdot \sum_{i=1}^{3}\left(\frac{d x_{i}}{d t}\right)^{2}
$$

This model leads to Newton's equations. In modern physics, most physical laws are formulated in terms of the Principle of Least Action, according to which the trajectory is selected in such a way that the action $S=\int L d t$ is the smallest possible. In particular, for Newtonian physics, the exact same expression $S$ - as we came up with based on fuzzy methodology - leads exactly to Newton's laws.

Comment. With the fuzzy approach, we not only get the most reasonable Newton's trajectory, we also get the degree $\exp (-k \cdot S)$ with which all other trajectories are reasonable. In Newton's physics, only one trajectory is possible, but in quantum physics, non-Newtonian trajectories are also possible, and the "amplitude" of each trajectory is determined by exactly this formula $\exp (-k \cdot S)$ - albeit with a complex value $k$. This fact makes the above derivation even more interesting.

\section{Beyond the Simplest Netwon's Equations}

Need to go beyond Newton's equations. In our analysis of the Newton's equations, we assume that the expression for the potential energy $V(x)$ is given. However, in reality, this expression also needs to be determined. The potential energy represents a field - e.g., electrostatic, gravitational, etc. - so, in addition to mechanics, we must also find the equations that describe the corresponding field.

Gravitational field: main idea. Let us consider the simplest case of a gravitational field. We will consider it in the Newtonian approximation, where it is described by a scalar function $V(x)$.

The main physical property of the gravitational field is that it changes very slowly: gravitational pull of the Earth, for example, is caused by the Earth as a whole, so if we move a little bit, we still feel approximately the same gravitation. It is a known empirical fact that the differences in the gravitational field at different earth locations are very small (but, by the way, very important for geophysics, because they provide a good overall understanding of what is located below the Earth surface).

Thus, all the components $\partial V \partial x_{i}$ of the gradient of the gravitational field must be small. This situation is similar to kinetic energy and different from potential energy in the sense that we want these values to be close to 0 . Similarly to the case of kinetic energy, this is equivalent to requiring that the squares of the derivatives be small.

Derivation of the resulting model. Thus, we arrive at the condition that for all locations $x$, all squares of partial derivatives must be small. For each location and for each $i$, the corresponding requirement that the square of the derivative is small can be described by the degree

$$
\exp \left(-k \cdot\left(\frac{\partial V}{\partial x_{i}}\right)^{2}\right)
$$

By using the product t-norm to combine these values, we get the expression

$$
\prod_{x} \prod_{i=1}^{3} \exp \left(-k \cdot\left(\frac{\partial V}{\partial x_{i}}\right)^{2}\right)
$$

As in the Newton's case, this expression can be represented as $\exp (-k \cdot S)$, where

$$
S=\sum_{x} \sum_{i=1}^{3}\left(\frac{\partial V}{\partial x_{i}}\right)^{2}
$$


Taking into account that we have infinitely many spatial locations $x$, we get an integral instead of the sum: $S=\int L d x$, where

$$
L(x)=\sum_{i=1}^{3}\left(\frac{\partial V}{\partial x_{i}}\right)^{2}
$$

This model leads to Newton's formulas for the gravitation force. It is known that minimizing this expression leads to the equation

$$
\sum_{i=1}^{3} \frac{\partial^{2} V}{\partial x_{i}^{2}}=0
$$

that leads to Newton's gravitational potential

$$
V(x) \sim \frac{1}{r}
$$

that, in turns, leads to the known expression for the gravitational force $F \sim r^{-2}$.

Comment. Similar arguments can lead to other known action principles and thus, to other fundamental physical equations.

Discussion. At present, this is just a theoretical exercise/proof of concept, whose main objective is to provide one more validation for the existing fuzzy methodology.

However, the fact that the existing fuzzy methodology enables us to transform informal ("fuzzy") description of physical phenomena into well-known physical equations makes us hope that in the future, when new physical phenomena will be discovered, fuzzy methodology may help generate the equations describing these phenomena.

\section{ACKNOWLEDGMENTS}

This work was supported in part by the National Science Foundation grants HRD-0734825 and DUE-0926721, by Grant 1 T36 GM078000-01 from the National Institutes of Health, by Grant MSM 6198898701 from MŠMT of Czech Republic, and by Grant 5015 "Application of fuzzy logic with operators in the knowledge based systems" from the Science and Technology Centre in Ukraine (STCU), funded by European Union.

The authors are thankful to the anonymous referees for valuable suggestions.

\section{REFERENCES}

[1] J. Aczel, Lectures on Functional Equations and Their Applications, Dover Publ., New York, 2006.

[2] R. Feynman, R. Leighton, and M. Sands, The Feynman Lectures on Physics, Addison Wesley, Boston, Massachusetts, 2005.

[3] G. Klir and B. Yuan, Fuzzy Sets and Fuzzy Logic: Theory and Applications, Upper Saddle River, New Jersey: Prentice Hall, 1995.

[4] H. T. Nguyen and E. A. Walker, First Course on Fuzzy Logic, CRC Press, Boca Raton, Florida, 2006.

\section{APPENDIX: VARIATIONAL EQUATIONS}

General derivation. Let us recall how we can transform the Least Action Principle into a differential equation. Let us first do it on the example on Newton-type situation, where we need to find a function $x(t)$ that minimizes the following expression:

$$
S=\int L(x, \dot{x}) d t \rightarrow \min .
$$

Minimizing means, in particular, that if we take any function $\Delta x(t)$ and consider a function $S(\alpha)=x+\alpha \cdot \Delta x$, then this function must attain its maximum for $\alpha=0$. Thus, the derivative of $S(\alpha)$ at $\alpha=0$ must be 0 . Differentiating the expression

$$
S(\alpha)=\int L(x+\alpha \cdot \Delta x, \dot{x}+\alpha \cdot \Delta \dot{x}) d t
$$

and equating the derivative to 0 , we conclude that

$$
\begin{gathered}
\int\left(\frac{\partial L}{\partial x} \cdot \Delta x+\frac{\partial L}{\partial \dot{x}} \cdot \Delta \dot{x}\right) d t= \\
\int\left(\frac{\partial L}{\partial x} \cdot \Delta x\right) d t+\int\left(\frac{\partial L}{\partial \dot{x}} \cdot \Delta \dot{x}\right) d t=0 .
\end{gathered}
$$

Integrating the second term by parts, we conclude that

$$
\int\left(\frac{\partial L}{\partial x}-\frac{d}{d t}\left(\frac{\partial L}{\partial \dot{x}}\right)\right) \cdot \Delta x d t=0 .
$$

This must be true for every function $\Delta x(t)$, in particular for a function that is equal to 0 everywhere except for a small vicinity of a moment $t$, For this function, the integral is proportional to the value of the expression

$$
\frac{\partial L}{\partial x}-\frac{d}{d t}\left(\frac{\partial L}{\partial \dot{x}}\right)
$$

at the point $t$. Since the integral is 0 , this expression must also be equal to 0 :

$$
\frac{\partial L}{\partial x}-\frac{d}{d t}\left(\frac{\partial L}{\partial \dot{x}}\right)=0 .
$$

The resulting equations are known as Euler-Lagrange equations.

Case of Newton's laws. In particular, for the Newton's case, when

$$
L=V(x)-\frac{1}{2} \cdot m \cdot \sum_{i=1}^{3}\left(\frac{d x_{i}}{d t}\right)^{2},
$$

for each of the components $x_{i}(t)$, we have

$$
\frac{\partial L}{\partial x_{i}}=\frac{\partial V}{\partial x_{i}}
$$

and

$$
\frac{\partial L}{\partial \dot{x}_{i}}=-m \cdot \frac{d x_{i}}{d t} .
$$

Thus, Euler-Lagrange's equations lead to

$$
\frac{\partial V}{\partial x}+m \cdot \frac{d}{d t}\left(\frac{d x_{i}}{d t}\right)=0
$$


i.e., to Newton's equations

$$
m \cdot \frac{d^{2} x_{i}}{d t^{2}}=-\frac{\partial V}{\partial x_{i}} .
$$$$
\frac{\partial L}{\partial \varphi}-\sum_{i=1}^{3} \frac{\partial}{\partial x_{i}}\left(\frac{\partial L}{\partial \varphi_{, i}}\right)=0
$$

where

General case. In the general case, Euler-Lagrange equations take the form

$$
\varphi, \stackrel{\text { def }}{=} \frac{\partial \varphi}{\partial x_{i}}
$$

